\title{
FM Model Based Fingerprint Reconstruction from Minutiae Template
}

\author{
Jianjiang Feng and Anil K. Jain \\ Department of Computer Science and Engineering \\ Michigan State University \\ $\{j$ feng, jain\}@cse.msu.edu
}

\begin{abstract}
Minutiae-based representation is the most widely adopted fingerprint representation scheme. The compactness of minutiae template has created an impression that the minutiae template does not contain sufficient information to allow the reconstruction of the original fingerprint image. This belief has now been shown to be false; several algorithms have been proposed that can reconstruct fingerprint images from minutiae templates. However, these reconstruction techniques have a common weak point: many spurious minutiae, not included in the original minutiae template, are generated in the reconstructed image. Moreover, some of these techniques can only reconstruct a partial fingerprint. In this paper, a novel fingerprint reconstruction algorithm is proposed, which not only reconstructs the whole fingerprint, but the reconstructed fingerprint contains very few spurious minutiae. A fingerprint image is modeled as a 2D Frequency Modulation (FM) signal whose phase consists of the continuous part and the spiral part (which corresponds to minutiae). An algorithm is proposed to reconstruct the continuous phase from minutiae. The proposed reconstruction algorithm has been evaluated with respect to the success rates of type-I attack (match the reconstructed fingerprint against the original fingerprint) and type-II attack (match the reconstructed fingerprint against the different impressions of the original fingerprint) using a commercial fingerprint recognition system. Both types of attacks were shown to be successful in deceiving the fingerprint system.
\end{abstract}

Keywords: Fingerprint synthesis, fingerprint reconstruction, minutiae, AM-FM, orientation field.

\section{Introduction}

Fingerprint recognition systems play a crucial role in many situations where a person needs to be verified or identified with high confidence. A fingerprint is an oriented texture consisting of interleaving ridges and valleys. The pattern of minutiae, which are the endpoints and merging points of ridges, is believed to be unique to each finger. Due to its distinctiveness and compactness, minutiae-based representation has become the most widely adopted fingerprint representation scheme. Since minutiae template is a compact representation of a fingerprint, it has been assumed that it is not possible to reconstruct the original fingerprint from a minutiae template. In other words, minutiae extraction algorithms have been traditionally viewed as a "one-way" transform. Recently, however, this belief has been challenged by some researchers [1, 2, 3] who were successful in reconstructing a fingerprint image from the given minutiae template. 
Hill [1] proposed a technique to determine the fingerprint structure from minutiae template (including singular points). In this work, orientation field was generated based on singular points according to the model in [4]. A line drawing algorithm was used to generate a sequence of splines passing through the minutiae. Ross et al. [2] first estimated the orientation field using selected minutiae triplets in the template. Streamlines were then traced starting from minutiae and border points. Linear Integral Convolution was used to impart texture-like appearance to the ridges. Finally, the image was smoothed to obtain wider ridges. This reconstruction algorithm can only generate a partial fingerprint. In addition, streamlines that terminated due to distance constraint between adjacent streamlines generate spurious minutiae. Cappelli et al. [3] estimated the orientation field by fitting a modified model proposed in [5] to the minutiae directions. Gabor filtering is iteratively performed starting from minutiae on an image initialized by local minutiae pattern. A rendering step is performed to make the reconstructed fingerprint image appear more realistic. However, this algorithm also generates many spurious minutiae in the reconstructed fingerprints.

Fingerprint reconstruction from minutiae (hereinafter simply referred to as fingerprint reconstruction) is very similar to fingerprint synthesis [6] except that the goals and the inputs of the two techniques are different. The goal of fingerprint reconstruction is to obtain an artificial fingerprint that resembles the original fingerprint as much as possible, while the goal of fingerprint synthesis is to generate artificial fingerprints that are as realistic as possible. For fingerprint reconstruction, the minutiae from a given fingerprint must be provided, while for fingerprint synthesis, no input is needed (except for statistical models learned from many real fingerprints).

The well-known SFINGE fingerprint synthesis method of Cappelli et al. [6] performs Gabor filtering on a seed image according to the orientation and frequency images; minutiae automatically emerge during the filtering procedure. Some intraclass variations, such as spatial transformation, touching area, nonlinear distortion, ridge dilation/shrinking and noise, are simulated to generate realistic impressions of the master fingerprint. One main limitation of SFINGE is that minutiae can not be controlled. As a result, SFINGE may generate problematic fingerprints that contain too few minutiae or very long ridges. It is well known that the distribution of minutiae in fingerprints is not random and fingerprints of different pattern types have different minutiae distributions [2]. The minutiae distribution of fingerprints generated by SFINGE may not conform to such distributions since these minutiae are automatically generated during the image filtering process. Similar fingerprint synthesis methods have also been proposed in [7, 8]. Reaction-diffusion technique described in [9] can also be used for synthesizing fingerprints. Bicz [10] described a fingerprint synthesis technique based on 2D FM model. The phase of FM model consists of the continuous component and the spiral component, which corresponds to minutiae. A fingerprint is synthesized by generating each component separately and combining them. Separation of the continuous phase and the spiral phase makes minutiae controllable. However, the most important step, generating continuous phase component, was not described in [10]. According to the demo software provided by the author, only a partial fingerprint (around the core) can be generated and the orientation field of each of the four fingerprint patterns is fixed. Larkin and Fletcher [11] proposed a fingerprint analysis framework based on AM-FM 
model where a fingerprint image is decomposed into the four components of the model. Separate compression of each component leads to a compressed fingerprint with an impressive compression factor of 239.

In this paper, a novel approach to fingerprint reconstruction from minutiae template is proposed, which uses FM model as the fingerprint representation scheme. Specifically, a new algorithm is proposed to reconstruct the continuous phase of FM model from minutiae. The advantages of our approach over existing approaches to fingerprint reconstruction [1, 2, 3] are: (i) the complete fingerprint can be reconstructed, and (ii) the reconstructed fingerprint contains very few spurious minutiae. The proposed reconstruction algorithm has been quantitatively assessed by matching reconstructed fingerprints against the original fingerprints (termed as type-I attack) and against different impressions of the original fingerprints (termed as type-II attack) using a commercial fingerprint SDK, Neurotechnology VeriFinger 4.2 [12]. Type-I attack was found to have a high chance in deceiving the fingerprint recognition system in both the verification and identification experiments. Type-II attack also has a significantly higher accept rate than that of impostor match.

\section{Fingerprint Representation}

Larkin and Fletcher [11] proposed to represent a fingerprint image as a 2D amplitude and frequency modulated (AM-FM) signal:

$$
I(x, y)=a(x, y)+b(x, y) \cos (\Psi(x, y))+n(x, y),
$$

which is composed of four components: the intensity offset $a(x, y)$, the amplitude $b(x, y)$, the phase $\Psi(x, y)$, and the noise $n(x, y)$. Here we are only interested in the phase $\Psi(x, y)$, since ridges and minutiae are totally determined by the phase; the other three components just make the fingerprint appear realistic. Therefore, an ideal fingerprint is represented as a 2D FM signal:

$$
I(x, y)=\cos (\Psi(x, y)) .
$$

According to the Helmholtz Decomposition Theorem [13], the phase can be uniquely decomposed into two parts: the continuous phase and the spiral phase:

$$
\Psi(x, y)=\Psi_{C}(x, y)+\Psi_{S}(x, y) .
$$

The gradient of the continuous phase $\Psi_{C}(x, y)$ is termed as instantaneous frequency $G(x, y)$. The direction of instantaneous frequency is normal to ridge orientation. The amplitude of instantaneous frequency is equal to the ridge frequency.

The spiral phase $\Psi_{S}(x, y)$ corresponds to minutiae:

$$
\Psi_{S}(x, y)=\sum_{n=1}^{N} p_{n} \arctan \left(\frac{y-y_{n}}{x-x_{n}}\right),
$$

where $x_{n}$ and $y_{n}$ denote the coordinates of the $n$th minutia, and $N$ denotes the total number of minutiae. The direction of a minutia is determined by its polarity $p_{n} \in$ 


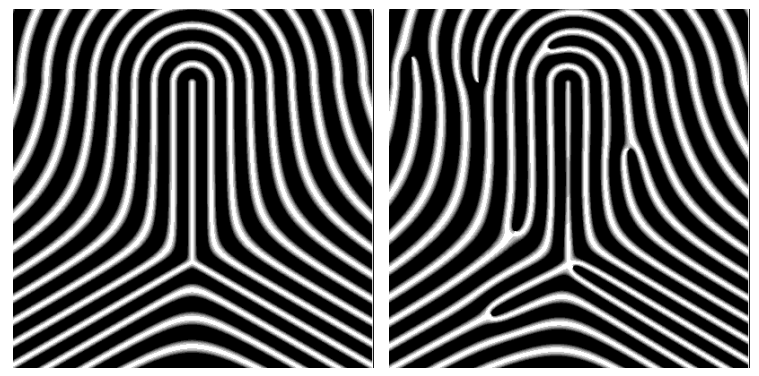

(a)

(b)

Fig. 1. (a) Synthetic fingerprint without minutiae and (b) synthetic fingerprint with 7 minutiae

$\{1,-1\}$ and the local ridge orientation $O\left(x_{n}, y_{n}\right)$, which is defined in the continuous phase. Assume the ridge orientation is in the range $[0, \pi]$. The direction of a minutia is equal to $O\left(x_{n}, y_{n}\right)$ when it has positive polarity, or $O\left(x_{n}, y_{n}\right)+\pi$ when it has negative polarity. Adding spiral to a continuous phase generates minutiae. Figure 1(a) shows a synthesized fingerprint that only contains continuous phase. Adding 7 spirals to the continuous phase leads to the synthesized fingerprint in Fig. 1(b)

\section{Fingerprint Reconstruction}

\subsection{Problem Statement}

A set of $N$ minutiae $\left\{x_{n}, y_{n}, \alpha_{n}\right\}, 1 \leq n \leq N$ of a fingerprint is given, where $\left(x_{n}, y_{n}\right)$ and $\alpha_{n}$ denote the location and direction of the $n$th minutia, respectively. In terms of FM model, the spiral phase is given and the direction of instantaneous frequency of the continuous phase is known at the locations of the $N$ minutiae. The problem is to reconstruct the original fingerprint image modeled by Eq. (1). This is obviously an ill-posed problem, since the important information required to reconstruct the continuous phase of fingerprints, ridge frequency, is unknown. Information needed to reconstruct realistic fingerprints, such as brightness, contrast, the background noise of fingerprint sensor, and detailed ridge features (pores, contours) etc., is also not available. A more practical goal is to estimate the FM representation of the original fingerprint, $\cos (\Psi(x, y))$. To obtain the phase $\Psi(x, y)$, the following three steps are performed: orientation field reconstruction, continuous phase reconstruction, and combination of the spiral phase and the continuous phase. The flow chart of the proposed fingerprint reconstruction algorithm is depicted in Fig. 2

\subsection{Orientation Field Reconstruction}

Ross et al. [2] used selected minutiae triplets to estimate the orientation field in triangles. Cappelli et al. [3] estimated orientation field by fitting an orientation field model to the orientations at minutiae. Both these methods have a minimum requirement on the number of minutiae. We propose a novel orientation field reconstruction algorithm that 


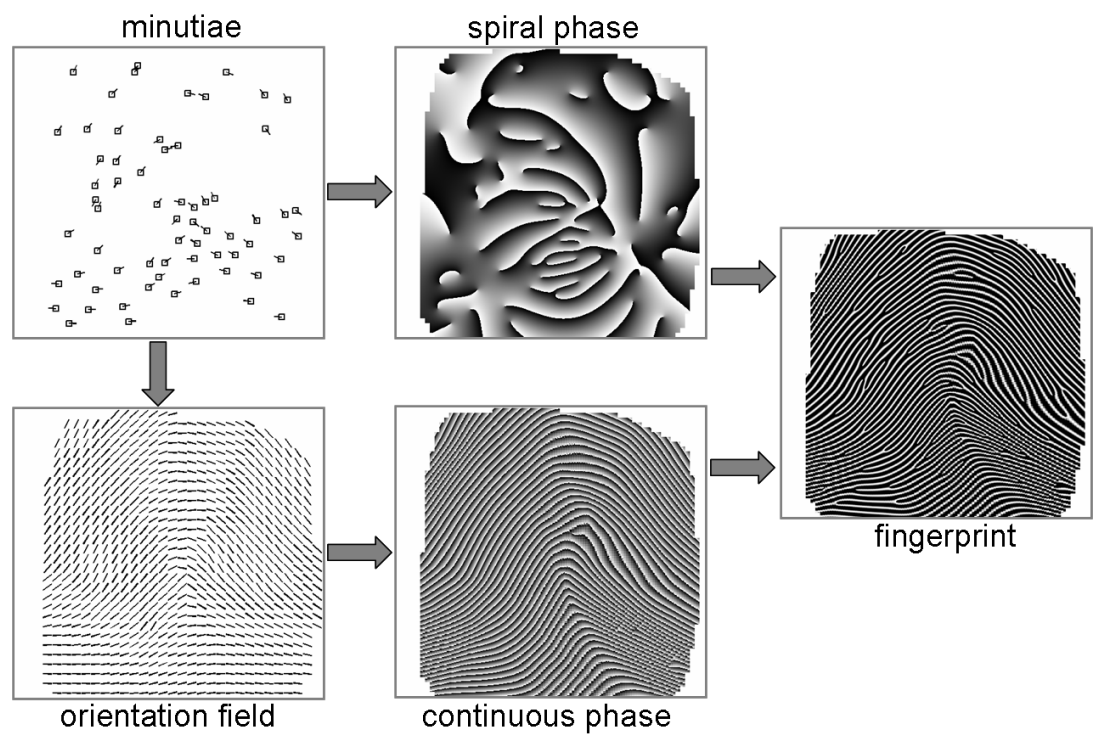

Fig. 2. Flow chart of the proposed fingerprint reconstruction algorithm

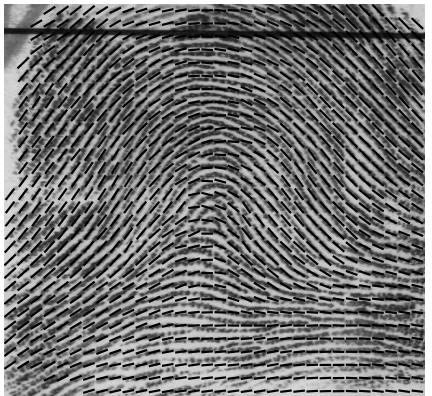

(a)

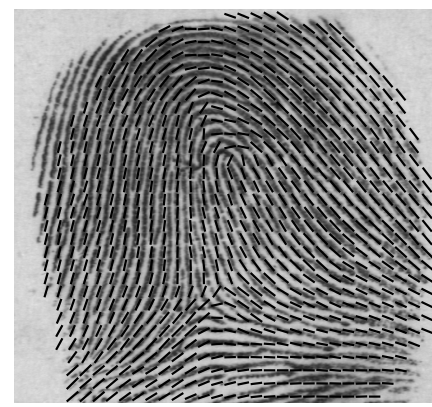

(c)

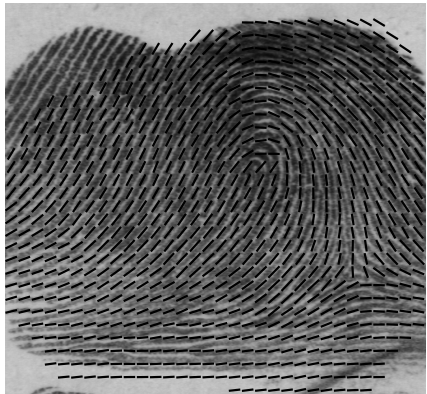

(b)

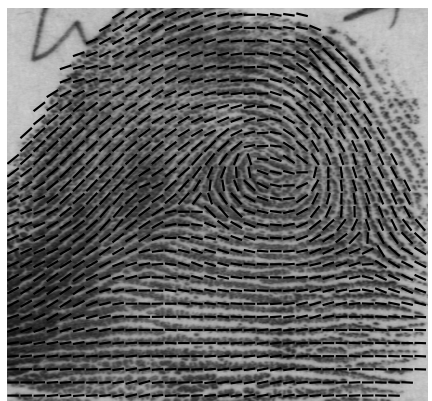

(d)

Fig. 3. Reconstructed orientation field for the four main types of fingerprints: (a) arch, (b) left loop, (c) right loop, and (d) whorl 


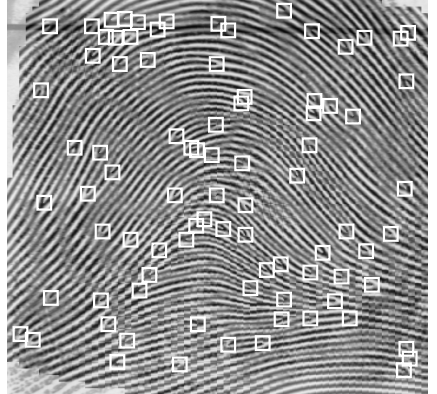

(a)

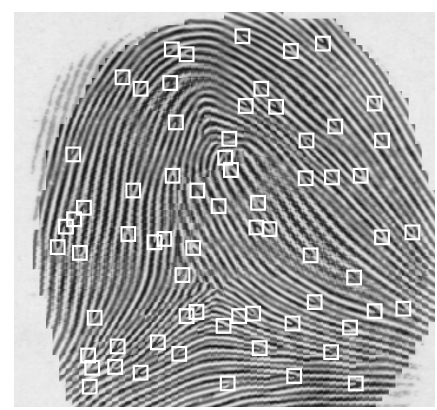

(c)

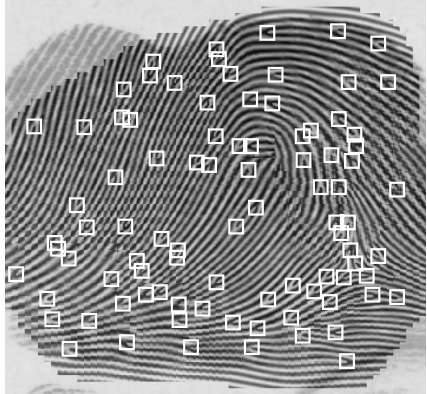

(b)

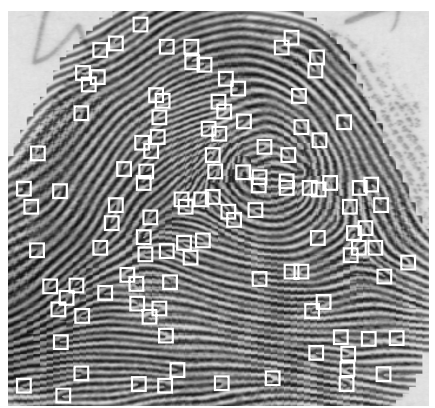

(d)

Fig. 4. Reconstructed fingerprints (overlaid on the original fingerprints) for the four main types of fingerprints: (a) arch, (b) left loop, (c) right loop, and (d) whorl. The original minutiae are denoted by white squares.

can work even when only one minutia is available. Figure 3 shows the reconstructed orientation field for the four main types of fingerprints.

The image is divided into non-overlapping blocks of $8 \times 8$ pixels. Foreground mask for the fingerprint image is obtained by dilating the convex hull of minutiae using a diskshaped structuring element of $8 \times 8$ pixels. The local ridge orientation at block $(m, n)$ is predicted by using the nearest minutia in each of the 8 sectors. The minutia direction $\alpha_{k}$ is doubled to make $\alpha_{k}$ equivalent to $\alpha_{k}+\pi$. The cosine and sine components of $2 \alpha_{k}$ of all the $K$ selected minutiae are summed:

$$
u=\sum_{k=1}^{K} \frac{\cos \left(2 \alpha_{k}\right)}{d_{k}}, v=\sum_{k=1}^{K} \frac{\sin \left(2 \alpha_{k}\right)}{d_{k}},
$$

where $d_{k}$ denotes the Euclidean distance between the block center and the $k$ th minutia. Then the orientation at block $(m, n)$ is computed as: $O(m, n)=\frac{1}{2} \arctan \left(\frac{v}{u}\right)$.

\subsection{Continuous Phase Reconstruction}

The continuous phase of a fingerprint is modeled by piecewise planes at each foreground block $(m, n)$ of $8 \times 8$ pixels: 


$$
\begin{array}{r}
\Psi_{C}(x, y)=2 \pi \cdot F(m, n) \cdot(\cos (O(m, n)) x+\sin (O(m, n)) y)+P(m, n), \\
8(m-1) \leq x<8 m, 8(n-1) \leq y<8 n,
\end{array}
$$

where $F(m, n), O(m, n)$ and $P(m, n)$ denote the ridge frequency, the ridge orientation and the phase offset at block $(m, n)$, respectively. Since it is not possible to estimate the ridge frequency from minutiae (if the ridge count information between minutiae is provided, then it is possible to estimate the ridge frequency), we have used a constant frequency value 0.1 for the whole image, which corresponds to a ridge period of 10 pixels in 500 ppi images. The only unknown value in Eq. (6), the phase offset $P(m, n)$, is estimated by the following algorithm.

Starting with a queue containing the top left-most block (whose phase offset is assumed to be 0), in each iteration, a block is obtained from the queue and each of its four-connected neighbors is checked if it has been reconstructed (namely, the phase offset has been estimated). If one of the neighboring blocks has not been reconstructed, the phase offset of this block is estimated and it is put into the queue. This procedure is performed until the queue is empty (which means that the continuous phase has been reconstructed at all the foreground blocks). An ancillary image is used to record the reconstructed blocks.

Here we describe how to estimate the phase offset of a block using all of the already reconstructed four-connected neighbors. Consider one of the neighbors, say block $(m-1, n)$, of block $(m, n)$. The phase images of these two blocks are required to be continuous at the border pixels $\{(x, y): x=8(m-1), 8(n-1) \leq y<8 n\}$. For each border pixel $(x, y)$, a phase offset of block $(m, n)$ is estimated:

$$
\begin{array}{r}
\psi=0.2 \pi \cdot(\cos (O(m-1, n)) \cdot x+\sin (O(m-1, n)) \cdot y)+P(m-1, n) \\
-0.2 \pi \cdot(\cos (O(m, n)) \cdot x+\sin (O(m, n)) \cdot y) .
\end{array}
$$

The mean value is used as the phase offset of block $(m, n)$.

After the continuous phase is reconstructed, the reconstructed fingerprint can be obtained by combining the continuous phase and the spiral phase. In Fig. 4, the reconstructed fingerprint for each of the four types of fingerprints in Fig. 3 is overlaid on the original fingerprint. The reconstructed fingerprints match the original fingerprints well. But, the reconstructed fingerprints do contain a few spurious minutiae, especially in the region of singularity. The main cause for the spurious minutiae is the assumption of a fixed ridge frequency for the whole image, which is not true for real fingerprints.

\section{Experiments}

A reconstructed fingerprint may be used to attack the system that contains the original fingerprint template (termed as type-I attack) or the systems where the same finger has also been enrolled (termed as type-II attack). Such fingerprint recognition systems may work in the verification mode or in the identification mode. To evaluate the performance of the proposed reconstruction algorithm in these situations, VeriFinger 4.2 by Neurotechnology [12] was assumed to be the attacked fingerprint recognition system.

The verification experiment was performed on FVC2002 DB1_A [14], which consists of 100 fingers and 8 impressions per finger. Fingerprints were reconstructed from 


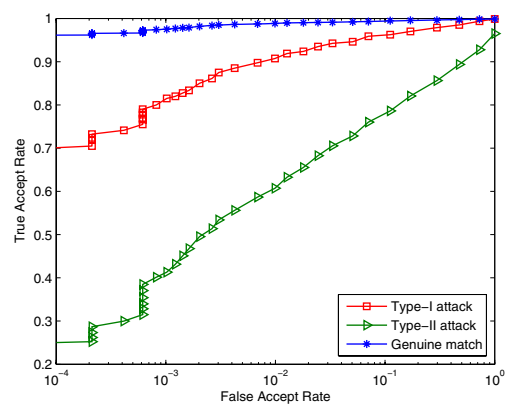

(a)

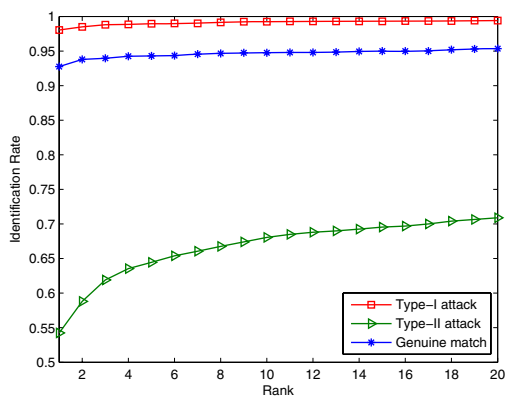

(b)

Fig. 5. Performance of the proposed reconstruction algorithm. (a) ROC curves of type-I attack, type-II attack and genuine match against impostor match on FVC2002 DB1_A. (b) CMC curves of type-I attack, type-II attack and genuine match on NIST SD4.

the minutiae templates of all the 800 images. Each reconstructed fingerprint was matched against all the 8 impressions of the same fingerprint in the database, which produced 800 type-I attacks and 5,600 type-II attacks. For the purpose of comparison, 2,800 genuine matches were obtained by cross matching 8 impressions of the same fingerprint and 4,950 impostor matches were obtained by cross matching the first impression of different fingerprints. By changing decision thresholds, the accept rates of type-I attack, type-II attack, genuine match and impostor match were obtained. The three Receiver Operating Characteristic (ROC) curves in Fig. 5(a) are plots of the true accept rates (termed as TAR) of type-I attack, type-II attack and genuine match against the false accept rates (termed as FAR) of impostor match. It can be observed that even under a very secure setting $(0.1 \%$ FAR), both type-I attack and type-II attack have a much higher accept rate ( $70 \%$ and $25 \%$, respectively) than the accept rate of impostor match, namely $0.1 \%$ FAR. As expected, type-I attack has more chance than type-II attack in deceiving a fingerprint verification system. But the impact of type-II atack should not be underestimated, since it can be launched against all fingerprint systems where the same finger has been enrolled. It should be noted that this performance can not be directly compared to the performance reported in [3], since (i) only type-I attack was considered in [3]; (ii) only the first impression (with large image size and good image quality) of each finger was used for reconstruction in [3]; and (iii) different fingerprint matchers have very different ability in dealing with artificial fingerprints, as shown in [3].

The identification experiment was performed on NIST SD4 [15], which consists of 2,000 pairs of ink-on-paper fingerprints (called file and search fingerprints, respectively). Fingerprints were reconstructed from the templates of 2,000 file fingerprints. The reconstructed fingerprints were matched against 2,000 file fingerprints and 2,000 search fingerprints to obtain 2,000 type-I attacks and 2,000 type-II attacks, respectively. For the purpose of comparison, 2,000 search fingerprints are also matched against 2,000 file fingerprints to obtain 2,000 genuine matches. The Cumulative Match Characteristic (CMC) curves of type-I attack, type-II attack and genuine match are given in Fig. 5(b). The rank-1 identification rate of $98.1 \%$ indicates that type-I attack has a very high 
identification rate in deceiving a fingerprint identification system, even higher than the identification rate of a genuine match. It should be noted that the rank-1 identification rate of type-I attack is only $23 \%$ in [2]. Since the same fingerprint database has been used and the same minutiae extraction and matching algorithms have been employed, we can conclude that the proposed reconstruction algorithm performs better than the algorithm of Ross et al. [2].

The reconstruction of fingerprints in FVC2002 DB1 and NIST SD4 takes around 3 and 8 seconds per image, respectively, when tested on a PC with 3GB of RAM and a $3 \mathrm{GHz}$ Pentium $4 \mathrm{CPU}$. The algorithm is currently implemented in MATLAB and we expect the computational costs to be significantly reduced after optimization.

\section{Conclusions and Future Work}

A FM model based fingerprint reconstruction scheme has been proposed. The phase of FM model is composed of the continuous phase and the spiral phase. A reconstructed fingerprint is obtained by reconstructing the orientation field, reconstructing the continuous phase and combining the continuous phase with the spiral phase. The experimental results show that the reconstructed images are very consistent with the original fingerprints and that there is a high chance to deceive a state-of-the-art commercial fingerprint recognition system.

The reconstructed fingerprints still contain a few spurious minutiae, especially in the high curvature regions. The spurious minutiae can be avoided by (i) detecting and subtracting spirals from the continuous phase, or (ii) permitting the ridge frequency to vary in a reasonable range. To obtain reconstructed images more consistent with the original fingerprints, ridge frequency and minutiae type should be utilized. To make the reconstructed fingerprints more realistic, brightness, ridge thickness, pores and noise should be modeled. The accept rate of the reconstructed fingerprints can be further improved by reducing the image quality around the spurious minutiae. To reduce the risk of attacks using reconstructed fingerprints, robust fingerprint template security [16] and spoof detection techniques [17] should be developed.

\section{Acknowledgments}

We would like to thank Dr. Kieran G. Larkin, Canon Information Systems Research Australia (CiSRA), for his help in generating the synthetic fingerprint in Fig. 1. This work was supported by ARO grant W911NF-06-1-0418 and NIJ grant 2007-RG-CX-K183.

\section{References}

1. Hill, C.: Risk of masquerade arising from the storage of biometrics. Master's thesis, Australian National University (2001)

2. Ross, A., Shah, J., Jain, A.K.: From template to image: Reconstructing fingerprints from minutiae points. IEEE Trans. Pattern Analysis and Machine Intelligence 29(4), 544-560 (2007) 
3. Cappelli, R., Lumini, A., Maio, D., Maltoni, D.: Fingerprint image reconstruction from standard templates. IEEE Trans. Pattern Analysis and Machine Intelligence 29(9), 1489-1503 (2007)

4. Sherlock, B.G., Monro, D.M.: A model for interpreting fingerprint topology. Pattern Recognition 26(7), 1047-1055 (1993)

5. Vizcaya, P.R., Gerhardt, L.A.: A nonlinear orientation model for global description of fingerprints. Pattern Recognition 29(7), 1221-1231 (1996)

6. Maltoni, D., Maio, D., Jain, A.K., Prabhakar, S.: Handbook of Fingerprint Recognition. Springer, Heidelberg (2003)

7. Novikov, S.O., Glushchenko, G.N.: Fingerprint ridges structure generation models. In: Proc. SPIE (International Workshop on Digital Image Processing and Computer Graphics), vol. 3346, pp. 270-274 (1997)

8. Araque, J.L., Baena, M., Chalela, B.E., Navarro, D., Vizcaya, P.R.: Synthesis of fingerprint images. In: Proc. 16th International Conference on Pattern Recognition, pp. 422-425 (2002)

9. Witkin, A., Kass, M.: Reaction-diffusion textures. SIGGRAPH Computer Graphics 25(4), 299-308 (1991)

10. Bicz, W.: The idea of description (reconstruction) of fingerprints with mathematical algorithms and history of the development of this idea at optel (2003),

http://www.optel.pl/article/english/idea.htm

11. Larkin, K.G., Fletcher, P.A.: A coherent framework for fingerprint analysis: are fingerprints holograms? Optics Express 15(14), 8667-8677 (2007)

12. Neurotechnology Inc., VeriFinger, http://www.neurotechnology.com

13. Ghiglia, D.C., Pritt, M.D.: Two-Dimensional Phase Unwrapping: Theory, Algorithms, and Software. John Wiley and Sons, New York (1998)

14. FVC 2002: The Second International Competition for Fingerprint Verification Algorithms, http: //bias.csr.unibo.it/fvc2002/

15. NIST SD4: NIST 8-Bit gray scale images of fingerprint image groups (FIGS), http://www.nist.gov/srd/nistsd4.htm

16. Nandakumar, K., Jain, A.K., Pankanti, S.: Fingerprint-based fuzzy vault: implementation and performance. IEEE Trans. Information Forensics and Security 2(4), 744-757 (2007)

17. Nixon, K.A., Rowe, R.K.: Multispectral fingerprint imaging for spoof detection. In: Proc. SPIE (Biometric Technology for Human Identification II), vol. 5779, pp. 214-225 (2005) 